\title{
CARBON DEPLETION IN CASE B MASS TRANSFER ALGOL-TYPE BINARIES
}

\author{
TAE S. YOON \\ Kyungpook National University \\ Dept. of Astronomy and Meteorology \\ Taegu 702-701 KOREA (South) \\ KENT HONEYCUTT \\ Indiana University \\ Astronomy Department \\ Bloomington, IN 47405 U.S.A.
}

\begin{abstract}
The surface carbon abundances of Algol secondaries are known to be low compared to field stars of similar type. The $\mathrm{C}$ and $\mathrm{N}$ anomalies of these $\mathrm{G}$ and $\mathrm{K}$ subgiants are undoubtedly due to the exposure of $\mathrm{CN}$ cycle processed material as mass transfer removes the outer layers of the star. Therefore the carbon abundance is a promising tool for helping fix the evolutionary state of Algols, particularly the amount of mass which has been lost from the secondary. We report here the carbon abundance of 12 Algol secondaries as deduced from the $\mathrm{g}$-band in spectra obtained during photometric totality. We compare the abundances to those from models of single stars of appropriate age and mass. The values of $\log \epsilon(\mathrm{C})$ for this sample fall $0.25-0.75$ dex below field $\mathrm{G}$ and $\mathrm{K}$ giants, and 1.25-1.75 dex above "stripped" single star models. This larger sample supports the conclusions of Parthasarthy et al. regarding mixing and mass loss, which they deduced from a smaller sample of carbon abundances.
\end{abstract}

\section{Introduction}

Carbon abundances of Algol-type binaries are available for only a few systems, and most are for the primary, not the secondary component (De Greve and Cugier 1989; Parthasarthy, Lambert and Tomkin 1983; Balachandran et al 1986). We report here 12 new determinations of $\log \epsilon(\mathrm{C})$ for Algol secondaries using the strength of the $\mathrm{g}$ band of the $\mathrm{CH}$ molecule. The 2.5 Angstrom resolution spectra were obtained at Kitt Peak using the IIDS spectrophotometer. The exposures consisted of successive 3-minute integrations, and the IIDS light curves permitted summing only those integrations which are actually in totality. The g-band equivalent widths were calibrated for $\log \epsilon(\mathrm{C})$ using observations of normal and $\mathrm{CH}$-weak $\mathrm{G}$ and $\mathrm{K}$ stars having published abundance analyses for carbon. 


\section{Comparison Values of Carbon Abundance}

A useful framework of comparison for the Algol carbon abundances is the carbon abundance from a single-star model of the appropriate age and mass. The age of the singlestar model is taken as the ending time of the phase of rapid mass transfer in the binary. The mass of the single star is taken as the initial mass of the secondary. The mass fraction in the single star is taken as the current mass of the secondary star in the binary. This IRS (Interior Radius of a Single Star) carbon abundance would be the observed abundance in the secondary star under the simple assumption that the secondary's evolution proceeds as single-star evolution up until the rapid phase of mass transfer, at which time the outer layers are removed, without mixing, down to the current mass.

Several expressions have been published which permit calculating an estimate of the final mass of the secondary, $\mathrm{M}_{2 f}$, from the initial mass of the secondary, $\mathrm{M}_{2 i}$ (Giuricin, Mardirossian and Mezzetti, 1983). We assume Case B mass transfer for our 12 stars because (with the possible exception of WW Cyg) published estimates of current mass give $M_{1}+M_{2} \leq 7 M_{o}$ (Giuricin et al., 1983). An average of all values obtained from the various $\mathrm{M}_{2 f}\left(\mathrm{M}_{2 i}\right)$ relations was used, along with $\mathrm{M}_{2 f}=0.5 \mathrm{M}_{2}$ (De Greve, 1986), to obtain the values of $\mathrm{M}_{2 i}$ in Table 1 .

An estimate of the ending time of the rapid phase of mass transfer is also needed to fix the IRS abundance. There are inconsistences in the ending time of the phase of rapid mass transfer from calculations of interacting binary star evolution (Kraicheva, 1987 and references therein), and the time in single star evolution for expansion into a giant. Therefore we adopt the time of the start of the hydrogen shell burning phase for case $B$ mass transfer to estimate the IRS carbon abundance, under the assumption that the duration of rapid mass transfer is short compared the duration of hydrogen shell burning.

Details on calculating the IRS carbon abundance can be found in Yoon (1991). Fortunately, the abundance is quite insensitive to both the ending time of the phase of rapid mass transfer and $\mathrm{M}_{2 i}$. The values of the carbon abundance at the IRS in Table 1 were taken from Iben's (Iben 1967, 1974 and references therein) single star evolutionary calculations. $\mathrm{M}_{2 i}$ for this sample is in the range 1.5-7 $\mathrm{M}_{o}$. In order to determine the carbon abundance at the chosen mass fraction, we assume that the general behavior of Iben's $3 \mathrm{M}_{o}$ model between points 5 and 13 of Iben's tracks are representative for this mass range. In any case the variation of composition with mass fraction is nearly independent of mass for the parameters of interest, as also mentioned by Parthasarathy et al. 1983 and by De Greve and Cugier 1989 .

Other carbon abundance comparisons of interest are the solar value of 8.67 (Lambert 1979 ) and the value for field $\mathrm{G}$ and $\mathrm{K}$ giants of 8.43 (Lambert and Ries 1981). The observed abundances in Table 1 have an estimated crror of 0.15 dex. 


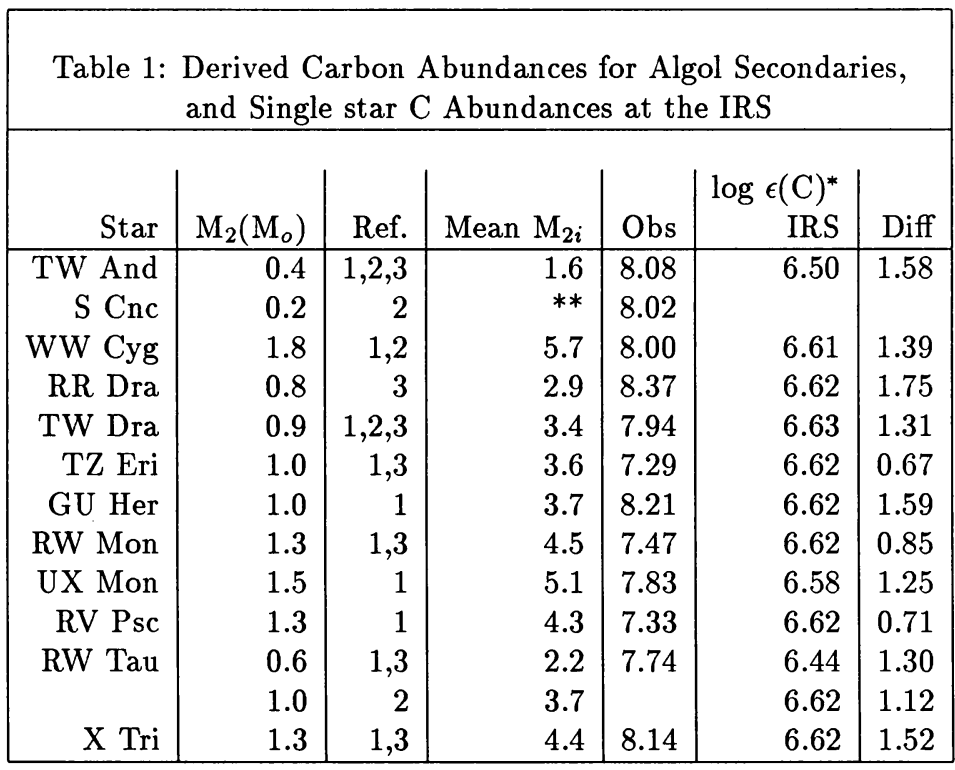

* logarithmic carbon on a scale for which the number abundance of hydrogen is 12 .

** $\mathrm{M}_{2 f}=0.5 \mathrm{M}_{2}$ probably not a valid assumption

$1=$ Brancewicz and Dworak 1980

$2=$ Giuricin, Mardirossian and Mezzetti 1983

$3=$ Budding 1984

\section{Discussion}

The observed carbon abundances in Table 1 are all much larger than the IRS abundance, indicating that any mass loss occuring prior to mixing did not exhaust the unprocessed envelope. The observed carbon abundances are all smaller than the value for field $\mathrm{G}$ and $\mathrm{K}$ giants which have undergone convective mixing of $\mathrm{CN}$ processed material with the full unprocessed envelope. This indicates that some appreciable mass loss of the envelope occured prior to convective mixing. These same conclusions were reached by Pathasarathy et al. 1983 on the basis of carbon abundances for three Algol secondaries. This larger data base supports this initial picture and refines our knowledge of the range of carbon abundances found in these stars. The column of differences in Table 1 was examined for possible dependencies on a variety of observed and derived parameters of the systems, but no convincing systematic behavior was revealed. Since mass loss subsequent to mixing will not change the surface column abundances, the variety of $\log \epsilon(\mathrm{C})$ may represent varying amounts of mass which has been lost after convective mixing developed. 


\section{Acknowlegements}

TSY would like to thank Dr. De Greve for useful comments and the Department of Education of Korea for financial help to attend this meeting. This work was partially sponsored by the National Science Foundation of the U.S.A.

\section{$5 \quad$ References}

Balachandran, S., Lambert, D.L., Tomkin, J. and Parthasarathy, M. 1986, M.N.R.A.S. $219,479$.

Brancewicz, H.K., and Dworak, T.Z. 1980, Acta Astron. 30, 501.

Budding, E. 1984, Bull. Info. CDS. No. 27.

De Greve, J.P. and Cugier 1989, Astron. Ap. 211, 356.

Giuricin, G., Mardirossian, F. and Mezzetti, M. 1983, Ap. J. Suppl. 52, 35.

Iben, I. 1967, Ann. Rev. Astron. Ap. 5, 571.

Iben, I. 1974, Ann. Rev. Astron. Ap. 12, 215.

Kraicheva, Z.T. 1987, Nauchn. Informatssi, 63, 105.

Lambert, D.L. 1979, M.N.R.A.S. 182, 249.

Lambert, D.L. and Ries, L.M. 1981, Ap. J. 248, 228.

Parthasarathy, M., Lambert, D.L. and Tomkin, J. 1983, M.N.R.A.S. 203, 1063.

Yoon, T.S. 1991, Ph.D. dissertation, Indiana University 